\title{
ON A HOMOTOPY CLASSIFICATION OF MAPPINGS \\ OF AN $(n+1)$ DIMENSIONAL COMPLEX INTO AN \\ ARCWISE CONNECTED TOPOLOGICAL SPACE WHICH \\ IS ASPHERICAL IN DIMENSIONS LESS THAN $n(n>2)$
}

\author{
NOBUO SHIMADA and HIROSHI UEHARA
}

Pontrjagin classified mappings of a three dimensional sphere into an $n$ dimensional complex, where he made use of a new type of product of cocycles. By the aid of the generalized Pontrjagin's product of cocycles Steenrod enumerated effectively all the homotopy classes of mappings of an $(n+1)$ dimensional complex into an $n$ sphere. According to the recent issue of the Mathematical Reviews it is reported that M. M. Postnikov extended Steenrod's case to the case where an arcwise connected topological space which is aspherical in dimensions less than $n$, takes place of an $n$ sphere. (Postnikov M. M., Classification of continuous mappings of an $(n+1)$ dimensional complex into a connected topological space which is aspherical in dimensions less than $n$. Doklady Akad. Nauk SSSR (N.S.) 71., 1027-1028, 1950 (Russian. No. proof is given.)) But here in Japan no details are yet to hand. We intend to give a solution to this problem in case where $n>2$, and also to give an application concerning the $(n$ $+3)$-extension cocycle.

$\S 1$. The simplest case where the $n$-th homotopy group $\pi_{n}(Y)$ of $Y$ has a finite base, each element of which is not of finite order.

Let $X$ be a finite complex with a fixed decomposition and let $Y$ be an arcwise connected topological space aspherical in dimensions less than $n .\left\{\alpha_{i} ; i=1\right.$, $\ldots, \lambda\}$ denotes a base of $\pi_{n}(Y)$ and a mapping $h_{i}: S^{n} \rightarrow Y(i=1, \ldots, \lambda)$ represents $\alpha_{i}$. Let $\eta: S^{n+1} \rightarrow S^{n}$ be a mapping, which represents the generator $\beta$ of $\pi_{n+1}\left(S^{n}\right) . \quad\left(h_{i} \eta\right)$ denotes the element of $\pi_{n+1}(Y)$ which is represented by a mapping $h_{i} \eta: S^{n+1} \rightarrow Y$. Now two groups $\pi_{n}(Y)$ and $\pi_{n}(Y)$ form a group pair with respect to $\pi_{n+1}(Y)$ when we define

i) $\quad \alpha_{i} \circ \alpha_{j}=0$, where $i \neq j$, and 0 is the unity of $\pi_{n+1}(Y)$,

ii) $\quad \alpha_{i} \circ \alpha_{i}=\left(h_{i} \eta\right)$,

iii) the bilinearity is assumed with respect to operation.

Received April 16, 1951. 
Then for two elements $\alpha, \beta \in \pi_{n}(Y)$, where $\alpha=\sum_{i=1}^{\lambda} a_{i} \alpha_{i}, \beta=\sum_{i=1}^{\lambda} b_{i} \alpha_{i}, \alpha \circ \beta$ is uniquely determined to be an element of $\pi_{n+1}(Y)$;

$$
\alpha \circ \beta=\left(\sum_{i=1}^{\lambda} a_{i} \alpha_{i}\right) \circ\left(\sum_{j=1}^{\lambda} b_{j} \alpha_{j}\right)=\sum_{i, j}\left(a_{i} b_{j}\right) \alpha_{i} \circ \alpha_{j}=\sum_{i=1}^{\lambda}\left(a_{i} b_{i}\right)\left(h_{i} \eta\right) \in \pi_{n+1}(Y) .
$$

We have the following properties concerning o operation.

$$
\begin{aligned}
& \alpha \circ \beta=\beta \circ \alpha \\
& \alpha \circ(\beta+\gamma)=\alpha \circ \beta+\alpha \circ \gamma \\
& 2 \alpha \circ \beta=0 .
\end{aligned}
$$

As was shown by Steenrod [2], a cell complex $M_{i}^{n+2}(i=1, \ldots, \lambda)$ is constructed by suspension, starting with a two dimensional complex projective plane $M^{4} . M_{i}^{n+2}(i=1, \ldots, \lambda)$ is decomposed into three cells : an $(n+2)$ dimensional cell $E_{i}{ }^{n+2}$, an $n$ dimensional cell $E_{i}^{n}$, and a point $E^{0}$. Steenrod showed $\left\{E_{i}{ }^{n}\right\} \bigcup_{n=-}$ $\left\{E_{i}{ }^{n}\right\}=\left\{E_{i}{ }^{n+2}\right\}$. Let $M^{n+2}$ be a cell complex $M_{1}{ }^{n+2} \vee M_{2}{ }^{n+2} \vee \ldots \vee M_{\lambda}{ }^{n+2}$ which is an union of $M_{i}{ }^{n+2}(i=1, \ldots, \lambda)$, joined together at a point $E^{0}=E_{1}{ }^{0}=\ldots=E_{\lambda}{ }^{0}$. Then it is easily seen that $\left\{E_{i}^{n}\right\} \bigcup_{n-2}\left\{E_{i}^{n}\right\}=\left\{E_{i}^{n+2}\right\}$ and $\left\{E_{i}^{n}\right\} \bigcup_{n-2}\left\{E_{j}^{n}\right\}=0(i \neq j)$. Thus we define that $E_{i}^{n} \bigcup_{n=2} E_{i}^{n}=E_{i}^{n+2}$ and $E_{i}^{n} \bigcup_{n=2} E_{j}^{n}=0 \quad(i \neq i)$. Let $s_{i}^{n}$ be a cocyle $\alpha_{i} E_{i}^{n}$ of $M^{n+2}$ and let $s_{i}^{n+2}$ be a cocycle $\left(h_{i} \eta\right) E_{i}^{n+2}$ of $M^{n+2}$, then we have

$$
\begin{aligned}
s_{i}^{n} \bigcup_{n-2} s_{i}^{n} & =s_{i}^{n+2} \\
s_{i}^{n} \bigcup_{n-2} s_{j}^{n} & =0 \quad(i \neq j) .
\end{aligned}
$$

Let $f$ be a mapping of $X^{n}$ into $Y$ such that $f\left(X^{n-1}\right)=*$, where $X^{r}$ is the $r$ skelton of a complex $X$ and $*$ is a fixed point of $Y$. Following Eilenberg [1], $C_{f}{ }^{n}$ denotes an $n$ cochain $\sum_{i} \theta_{i} \sigma_{i}{ }^{n}$ where the coefficient $\theta_{i}$ of an $n$ simplex $\sigma_{i}^{n}$ of $X$ is an element belonging to $\pi_{n}(Y, *)$ such that the mapping $f: \sigma_{i}{ }^{n} \rightarrow Y$ represents.

THEOREM 1. If $\delta C_{f}^{n}=0$, we have

$$
\text { the secondary obstruction }\left\{C^{n+2}(f)\right\}=\left\{C_{f}^{n}\right\} \bigcup_{n-2}\left\{C_{f^{n}}\right\} \text {. }
$$

Proof. Let $f\left(\sigma_{j}{ }^{n}\right)$ represent an element $\sum_{i=1}^{\lambda} m_{j i} \alpha_{i}$ of $\pi_{n}(Y)$. We construct a mapping $h: S_{1}{ }^{n} \vee \ldots \vee S_{\lambda}{ }^{n} \rightarrow Y$ such that $h\left(S_{i}{ }^{n}\right)$ represents $\alpha_{i}$ (for $i=1, \ldots$, $\lambda)$, where $S_{1}{ }^{n} \vee \ldots \vee S_{\lambda}{ }^{n}$ denotes, as is usually designated, a union of spheres $S_{i}^{n}(i=1, \ldots, \lambda)$ joined together at a point $E^{0}$. Again, defining a mapping $\psi: X^{n} \rightarrow S_{1}{ }^{n} \vee S_{2}{ }^{n} \vee \ldots \vee S_{\lambda}{ }^{n}$ such that $\psi$ maps boundary $\partial \sigma_{j}^{n}$ of $\sigma_{j}{ }^{n}$ (for any $j$ ) onto $E^{0}$ and maps $\sigma_{j}^{n}$ ontn $S_{i}^{n}$ with degree $m_{j i}$, we see that $f$ is homotopic to $h \psi$. Since $C^{n+2}(f)$ is cohomologous to $C^{n+2}(h \psi)$, we may assume $f=h \psi$ without loss of generality. As $C_{f}{ }^{n}$ is an $n$ cocyle and so $\delta C_{f^{n}}\left(\sigma_{k}{ }^{n+1}\right)=C_{f^{n}}\left(\partial \sigma_{k}{ }^{n+1}\right)$ 
$=h\left(\psi\left(\partial \sigma_{k}^{n+1}\right)\right)=h\left(\sum_{i=1}^{\lambda} a_{k i} S_{i}^{n}\right)=\left(\sum_{i=1}^{\lambda} a_{k i} \alpha_{i}=0\right.$, we have $a_{k i}=0$ for every $k$ and for $i=1, \ldots, \lambda$. Therefore $\psi$ can be extended to a mpping $\bar{\psi}: X^{n+1} \rightarrow S_{1}{ }^{n} \vee S_{2}{ }^{n} \vee$ $\ldots \vee S_{\lambda}{ }^{n}$. Let $\bar{f}$ be $h \bar{\psi}$, then we have $C^{n+2}(f)=C^{n+2}(\bar{f})$. As $\pi_{n+1}\left(S_{1}{ }^{n} \vee S_{2}{ }^{\lambda}\right.$ $\left.\vee \ldots \vee S_{\lambda}{ }^{n}\right)=\sum_{i=1}^{\lambda} \pi_{n+1}\left(S_{i}^{n}\right), \bar{\varphi}\left(\partial \sigma_{k}{ }^{n+2}\right)$ represents $\sum_{i=1}^{\lambda} \varepsilon_{k}{ }^{i} \beta_{i}$ where $\beta_{i}$ is the generator of $\pi_{n+1}\left(S^{n}\right)$ and $\varepsilon_{k}{ }^{i}$ is 1 or 0 according as $\bar{\psi}$ maps the boundary $\partial \sigma_{k}{ }^{n+2}$ of an $(n+2)$ simplex $\sigma_{k}{ }^{n+2}$ of $X$ into $S_{i}{ }^{n}$ essentially or not. Therefore it is easily verified that $C^{n+2}(f)\left(\sigma_{k}{ }^{n+2}\right)=\bar{f}\left(\partial \sigma_{k}^{n+2}\right)=h \bar{\psi}\left(\partial \sigma_{k}{ }^{n+2}\right)=\sum_{i=1}^{\lambda} \varepsilon_{k}{ }^{i}\left(h_{i} \eta\right)$. As was shown by Steenrod [2], $\bar{\psi}$ can be extended to a mapping $\overline{\bar{\psi}}: X^{n+2} \rightarrow M^{n+2}=M_{1}^{n+2} \vee \ldots$ $\vee M_{\lambda}^{n+2}$. Putting $s^{n+2}=\sum_{i=1}^{\lambda}\left(h_{i} \eta\right) E_{i}^{n+2}=\sum_{i=1}^{\lambda} s_{i}^{n+2}$ and $s^{n}=\sum_{i=1}^{\lambda} \alpha_{i} E_{i}^{n}=\sum_{i=1}^{\lambda} s_{i}$, we have $s^{n+2}=s^{n} \bigcup_{n-2} s^{n}$ from discussions referred to above.

$$
\begin{aligned}
\overline{\bar{\psi}}^{*} s^{n+2}\left(\sigma_{k}^{n+2}\right) & =\overline{\bar{\psi}}^{*}\left(\sum_{i=1}^{\lambda}\left(h_{i} \eta\right) E_{i}^{n+2}\right)\left(\sigma_{k}^{n+2}\right) \\
& =\left(\sum_{i=1}^{\lambda}\left(h_{i} \eta\right) E_{i}^{n+2}\right)\left(\overline{\bar{\psi}}\left(\sigma_{k}^{n+2}\right)\right)=\sum_{i=1}^{\lambda} \varepsilon_{k}^{i}\left(h_{i} \eta\right),
\end{aligned}
$$

so that we have

$$
\overline{\bar{\psi}}^{*} s^{n+2}=C^{n+2}(f) .
$$

Evidently $\overline{\bar{\psi}}^{*} s^{n+2}=\left(\overline{\bar{\psi}}^{*} s^{n}\right) \bigcup_{n-2}\left(\overline{\bar{\psi}}^{*} s^{n}\right)=\left(\bar{\psi}^{*} s^{n}\right) \bigcup_{n-2}\left(\bar{\psi}^{*} s^{n}\right)$ and $\left(\bar{\psi}^{*} s^{n}\right)\left(\sigma_{j}^{n}\right)=s^{n}\left(\bar{\psi}\left(\sigma_{j}^{n}\right)\right)$ $=s^{n}\left(\psi\left(\sigma_{j}^{n}\right)\right)=\sum_{i=1}^{\lambda} m_{j i} \alpha_{i}=C_{f^{n}}^{n}\left(\sigma_{j}^{n}\right)$, so that we have

$$
\overline{\bar{\psi}}^{*} s^{n+2}=C_{f}^{n} \bigcup_{n-2} C_{f}{ }^{n} \text {. }
$$

It follow that $\left\{C^{n+2}(f)\right\}=\left\{C_{f^{n}}\right\} \bigcup_{n-2}\left\{C_{f^{n}}\right\}$. This proves Theorem 1 .

$\S 2$ The cace where the $n$-th homotopy group $\pi_{n}(Y)$ of $Y$ is a cyclic group $\{\stackrel{m}{\alpha}\}$ of order $m$.

THEOREM 2. The analogous Theorem holds true in this second case.

Proof. Let $f\left(\sigma_{j}^{n}\right)$ represent an element $p_{j} \alpha$ and let a mapping $h: S^{n} \rightarrow Y$ represent the generator $\alpha$ of $\pi_{n}(Y)$. Define a mapping $\psi: X^{n} \rightarrow S^{n}$ such that $\psi\left(\sigma_{j}{ }^{n}\right)=p_{j} S^{n}$, then $f$ is homotopic to $h \psi$. It follows that $C_{f}{ }^{n}=C_{h \psi}{ }^{n}$ and $\left\{C^{n+2}(f)\right\}=\left\{C^{n+2}(h \psi)\right\}$. Without loss of generality it may be assumed that $f=h \psi$. As $C_{f}{ }^{n}$ is an $n$-cocycle and so $\delta C_{f}^{n}\left(\sigma_{j}{ }^{n+1}\right)=h\left(\psi\left(\partial \sigma_{j}{ }^{n+1}\right)\right)=h\left(q_{j} S^{n}\right)$ $=q_{j} \alpha=0$, we have $q_{j}= \pm r_{j} m$ where $r_{j}$ is an integer $\supseteqq 0$. Let $E^{n+1}$ be an $(n$ +1 ) element, whose boundary $\partial E^{n+1}$ is mapped onto $S^{n}$ with degree $m$. Then we designate by $E^{n+1} \cup S^{n}$ a cell complex which attaches $E^{n+1}$ to $S^{n}$, identifying the boundary point of $E^{n+1}$ with its image on $S^{n}$. If $r_{j}=0, \psi: \partial \sigma_{j}^{n+1} \rightarrow S^{n}$ can be extended to a mapping $\bar{\psi}: \sigma_{j}^{n+1} \rightarrow S^{n}$. In case $r_{j} \neq 0 \phi$ can be extended 
to a mapping $\bar{\Psi}: \sigma_{j}^{n+1} \rightarrow E^{n+1} \cup S^{n}$ in the following way. Dividing a simplex $\sigma_{j}^{n}=\left(a_{0}, a_{1}, \ldots, a_{n}\right)$ into $p_{j}$ parts by $(n-1)$ faces $a_{0} \Delta_{i}^{n-2}\left(i=1, \ldots,\left(p_{j}-1\right)\right)$, we may assume without loss of generality that $\psi$ maps $a_{0} \Delta_{i}^{n-2}$ into a point $E^{0}$ on $S^{n}\left(\varphi\left(\partial \sigma_{j}{ }^{n}\right)=E^{0}\right)$ and also each of $p_{j}$ subdivided $n$-simplexes onto $S^{n}$ with degree 1 . Then we can separate all the subdivided simplexes on $\partial \sigma_{j}^{n+1}$ into $r_{j}$ sets $\mathfrak{A}_{i}{ }^{j}=\left\{\tau_{i k}{ }^{j}\left(i=1, \ldots, r, k=1, \ldots, s_{i}\right)\right\}$ of simplexes such that the sum of degrees with which $\tau_{i k}{ }^{j}\left(k=1, \ldots, s_{i}\right)$ belonging to $\mathfrak{A}_{i}{ }^{j}$ are mapped onto $S^{n}$, is equal to $m$. Join $s_{i}$ simplexes belonging to $\mathfrak{A}_{i}^{j}$ by a tube $\Im_{i}{ }^{j}$ in the interior of $\sigma_{j}^{n+1}$ such that the tubes $\left\{\Im_{i}{ }^{j}(i=1, \ldots, r)\right\}$ do not intersect one another. Now $\psi$ can be extended such that $\psi$ maps $\partial \mathcal{S}_{i}^{j}$ into $E^{0}$. As the boundary $\not_{i}^{j}(i=1$, $\ldots, r)$ of the point set which consists of $\left\{\tau_{i k^{i}}\left(k=1, \ldots, s_{i}\right)\right\}$ and the tube $\Im_{i}^{j}$ is a topological $n$-sphere and $\psi$ maps $\oiint_{i}^{j}$ onts $S^{n}$ with degree $m$, it is seen that $\psi$ can be extended to a mapping $\bar{\psi}: \sigma_{j}^{n+1} \rightarrow E^{n+1} \cup S^{n}$ for such $j$ as $r_{j} \neq 0$. It follows that we have an extended mapping $\bar{\psi}: X^{n+1} \rightarrow E^{n+1} \cup S^{n}$ of $\psi$. Then it is easily verified that there is an extended mapping $\bar{h}: E^{n+1} \cup S^{n} \rightarrow Y$ of $h$. We designate by $\bar{f}$ an extended mapping $\bar{h} \cdot \bar{\psi}$ of $f$.

With respect to the closed subset $S^{n}$ of $E^{n+1} \cup S^{n}$, considering the homotopy sequence

$$
\longrightarrow \pi_{n+1}\left(S^{n}\right) \stackrel{i}{\rightarrow} \pi_{n+1}\left(E^{n+1} \cup S^{n}\right) \stackrel{j}{\rightarrow} \pi_{\dot{n}+1}\left(E^{n+1} \cup S^{n}, S^{n}\right) \stackrel{\partial}{\rightarrow} \pi_{n}\left(S^{n}\right) \longrightarrow
$$

it is easily seen that the boundary operation $\partial$ maps isomorphically $\pi_{n+1}\left(E^{n+1}\right.$ $\left.\cup S^{n}, S^{n}\right)$ into a subgroup $\{m k ; k=0, \pm 1, \ldots\}$ of $\pi_{n}\left(S^{n}\right)$, isomorphic to the integer group and that $\pi_{n+1}\left(S^{n}\right)(n>2)$, isomorphic to the cyclic group of order two, is mapped by $i$ onto $\pi_{n+1}\left(E^{n+1} \cup S^{n}\right)$. In case where $m$ is even, $i \eta: S^{n+1}$ $\stackrel{\eta}{\longrightarrow} S^{n} \stackrel{i}{\rightarrow} E^{n+1} \cup S^{n}$, (where $\eta$ represents the generator of $\pi_{n+1}\left(S^{n}\right)$ and $i$ the injection), represents the generator of $\pi_{n+1}\left(E^{n+1} \cup S^{n}\right)$, which is of order two, while if $m$ is odd, $\pi_{n+1}\left(E^{n+1} \cup S^{n}\right)$ is a trivial group.

Now $\bar{\psi}: \partial \sigma_{j}^{n+2} \rightarrow E^{n+1} \cup S^{n}$ is homotopic to $i \cdot \eta$ or to an inessential mapping. In the former case, taking an $(n+2)$ element $E_{j}{ }^{n+2}$ in the interior of $\sigma_{j}^{n+2}$, mapping $\partial E_{j}^{n+2}$ onto the closed subset $S^{n}$ of $E^{n+1} \cup S^{n}$ by $\eta$, we may have a homotopy between $\bar{\psi} \mid \partial \sigma_{j}{ }^{n+2}$ and $i \cdot \eta$ over $\sigma_{j}^{n+2}-E_{j}^{n+2}$ and also, with Steenrod, extend $\eta: \partial E_{j}^{n+2} \rightarrow S^{n}$ to a mapping $E_{j}^{n+2} \rightarrow M^{n+2} \supset S^{n}$. Therefore we have an extended mapping $\overline{\bar{\psi}}: X^{n+2} \rightarrow M^{n+2} \cup E^{n+1}$.

$$
C^{n+2}(f)\left(\sigma_{j}^{n+2}\right)=C^{n+2}(\bar{f})\left(\sigma_{j}^{n+2}\right)=\bar{h} \bar{\psi}\left(\partial \sigma_{j}^{n+2}\right)=\varepsilon(h \cdot \eta),
$$

where $(h \cdot \eta)$ is an element of $\pi_{n+1}(Y)$ represented by a mapping $h \eta: S^{n+1}$ $\stackrel{\eta}{\rightarrow} S^{n} \stackrel{n}{\rightarrow} Y$, and $\varepsilon$ is 1 or 0 according as $\bar{\psi}: \partial \sigma_{j}^{n+2} \rightarrow E^{n+1} \cup S^{n}$ is homotopic to 
in or to an inessential mapping. Now the following discussions are the same* as used in $\S 1$ and so omitted. It is concluded that $\left\{C^{n+2}(f)\right\}=\left\{C_{f^{n}}\right\} \bigcup_{n-2}\left\{C_{f^{n}}\right\}$.

§3. The most general case where $\pi_{n}(Y)$ has a countable infinite base.

Let $\left\{\alpha_{\lambda}:(\lambda=1,2, \ldots)\right\}$ be the elements of a base of $\pi_{n}(Y)$, whose orders are not finite and let $\left\{\beta_{\mu}:(\mu=1,2, \ldots)\right\}$ be the elements of a base, whose orders are $m_{\mu}(\mu=1,2, \ldots)$. Define a mapping $h:\left({S_{1}}^{n} \vee S_{2}{ }^{n} \vee \ldots\right) \vee\left(\bar{S}_{1}{ }^{n}\right.$ $\left.\vee \bar{S}_{2}{ }^{n} \vee \ldots\right) \rightarrow Y$ such that $h_{\lambda}=h \mid S_{\lambda}{ }^{n}$ and $\bar{h}_{\mu}=h \mid \bar{S}_{\mu}{ }^{n}$ represents $\alpha_{\lambda}$ and $\beta_{\mu}$ respectively. As discussed in $\S \S 1$. 2. we can define a mapping $\psi: X^{n} \rightarrow\left(S_{1}{ }^{n}\right.$ $\left.\vee S_{1}{ }^{n} \vee \ldots\right) \vee\left(\bar{S}_{1}{ }^{n} \vee \bar{S}_{1}{ }^{n} \vee \ldots\right)$ such that $f$ is homotopic to $h \cdot \psi$. Since $\delta C_{f^{n}}$ $=0$, we have $\psi\left(\partial \sigma_{j}{ }^{n+1}\right)=\sum_{i=1}^{k} \pm r_{i} m_{i} \bar{S}_{i}^{n}$, where $r_{i}$ is a integer $\supseteqq 0$, and all the spheres onto which $\sigma_{j}{ }^{n+1}$ is mapped by $\psi$ are finite and so are designated by $\bar{S}_{i}^{n}(i=1, \ldots, \kappa)$, changing the index. In virtue of arguments in $\S \S 1$. 2. we have an extended mapping $\bar{\psi}: X^{n+1} \rightarrow\left(S_{1}{ }^{n} \vee S_{2}{ }^{n} \vee \ldots \vee S_{\rho}{ }^{n}\right) \vee\left(P_{1}{ }^{n+1} \vee \ldots \vee P_{P^{\prime}}{ }^{n+1}\right)$, where $P_{i}^{n+1}=E_{i}{ }^{n+1} \cup \bar{S}_{i}{ }^{n}\left(i=1, \ldots, \rho^{\prime}\right)$, and also an extended mapping $\overline{\bar{h}}: P_{i}^{n+1}$ $\rightarrow Y$ of $\bar{h}_{i}$, so that $\bar{h}:\left({S_{1}}^{n} \vee \ldots \vee S_{p}{ }^{n}\right) \vee\left(P_{1}^{n+1} \vee \ldots \vee P_{p^{n+1}}\right) \rightarrow Y$ can be de. fined as an extension of $h$. Put $\bar{f}=\bar{\varphi} \bar{h}$. It is verified that $\pi_{n+1}\left(\left(S_{1}{ }^{n} \vee S_{2}{ }^{n}\right.\right.$ $\left.\left.\vee \ldots \vee S_{\mathrm{P}}{ }^{n}\right) \vee\left(P_{1}^{n+1} \vee \ldots \vee P_{\mathrm{P}^{\prime}}{ }^{n+1}\right)\right)=\sum_{i=1}^{p} \pi_{n+1}\left(S_{i}{ }^{n}\right)+\sum_{i=1}^{p^{\prime}} \pi_{n+1}\left(P_{i}^{n}\right)$ for $n>2$. Therefore $C^{n+2}(f)\left(\sigma_{j}^{n+2}\right)=C^{n+2}(\bar{f})\left(\partial \sigma_{j}^{n+2}\right)=\bar{h} \bar{\Psi}\left(\partial \sigma_{j}^{n+2}\right)=\sum_{i} \varepsilon_{i}\left(h_{i} \eta\right)+\sum_{j} \varepsilon_{j}\left(\bar{h}_{j} \eta\right)$. The later arguments are analogous as referred to in $\S \S 1$. 2. and so omitted. Now we have Theorem 3 , which furnishes an essential tool to the classification problem discussed in this paper.

THEOREM 3. In case where the $n$-th homotopy group of $Y$ has an infinite countable base, the analogous Theorem as Theorems 1. 2. holds true.

\section{§4. The classification of mappings of $X=X^{n+1}$ into $Y$.}

Because it is clear to classify mappings of $X^{n+1}$ into $Y$ now that Theorem 3 has been established, we shall give only the summary of this problem. Two mappings $f, g: X \rightarrow Y$ such that $f \mid X^{r}$ is homotopic to $g \mid X^{r}$ (for $r \leqq n+1$ ), are said to be $r$-homotopic each other. By the concept of $r$-homotopy, all the mappings of $X$ into $Y$ are divided into disjoint homotopy classes, which are referred to as $r$-homotopy classes. Then all the $n$-homotopy classes are in one to one correspondence with the $n$-th cohomology group $H_{n}(X)$ of $X$ because,

* In this case $E_{\imath}{ }^{n}$ is considered to be a cocyle modulo $m$, so that we have $\left\{\overline{1} E_{i}{ }^{n}\right\} \cup\left\{\bar{l} E_{n-2} E_{i}\right\}$ $=\left\{\overline{\overline{1}} E_{i}{ }^{n+2}\right\}$, where $\overline{1}, \overline{\overline{1}}$ are units of $I_{m}$ (cyclic group of order $m$ ), In respectively. Thus such a group pairing is the same as discussed in $\$ 1$. 
for any normal mapping $f$ belonging to an $n$-homotopy class $U^{\lambda}$, a cohomology class represented by a cocycle $C_{f^{n}}$ is uniquely determined, and moreover for any $n$-cocycle $C^{n}$ there exists a mapping $f: X \rightarrow Y$ such that $C_{f^{n}}=C^{n}$. Second$1 y$, the squareing homomorphism $S q_{n-3}: H_{n-1}\left(X, \pi_{n}(Y)\right) \rightarrow H_{n+1}\left(X, \pi_{n+1}(Y)\right)$ can be established (refer to Steenrod [2]). If we put $A_{n+1}(X)=S q_{n-3}\left(H_{n-1}(X\right.$, $\left.\pi_{n}(Y)\right)$ ), we have

THEOREM 4. For two normal mappings $f, g: X^{n+1} \rightarrow Y$ such that $f \mid X^{n}$ $=g \mid X^{n}, f$ is $(n+1)$-homotopic to $g$ if and only if $\left\{d^{n+1}(f, g)\right\}$ belongs to $A_{n+1}(X)$.

Proof. This theorem can be easily verified in an analogous way as Steenrod shows. The proofs of Theorem 4 and of the squaring homomorphism referred to above are accomplished essentially by the aid of Theorem 3 .

THEOREM 5. All the $(n+1)$-homotopy classes involved in an n-homotopy class are in one to one correspondence with $H_{n+1}\left(X, \pi_{n+1}(Y)\right) / S q_{n-3} H_{n-1}(X$, $\left.\pi_{n}(Y)\right)$.

\section{$\S 5$. The $(n+3)$-extension cocycle.}

Let $X$ be a finite simplicial complex and let $Y$ be an arcwise connected topological space whose homotopy groups vanish in dimensions less than $n$. Let $f, g$ be two maps of $X^{n+2}$ into $Y$ such that $f / X^{n}=g / X^{n}$. Denote the $(n+3)$-extension cocycles of $f, g$ with coefficients in $\pi_{n+2}(Y)$ by $c^{n+3}(f), c^{n+3}(g)$ respectively. Then we have

THEOREM 6.

$$
\begin{array}{ll}
c^{n+3}(f)-c^{n+3}(g) \backsim d^{n+1} \cup{ }_{n-1} d^{n+1} & (n>2), \\
c^{5}(f)-c^{5}(g) \backsim d^{3} \bigcup_{1} d^{3}+c^{2} \cup d^{3} & (n=2),
\end{array}
$$

where $d^{n+1}=d^{n+1}(f, g)$ is the $(n+1)$-difference cocycle with coefficients in $\pi_{n+1}(Y)$, and $c^{2}=c_{f}{ }^{2}=c_{g}{ }^{2}$ is the characteristic cocycle. The pairing of coefficients in the term $d^{n+1} \cup_{n-1} d^{n+1}$ is analogous as used in the previous sections, while the Whitehead product is taken as the pairing of coefficients in the term $c^{2} \cup d^{3}$.

\section{BIBLIOGRAPHY}

[1] S. Eilenberg, Cohomology and continuous mappings. Ann. of Math., 41 (1940), 231-251.

[2] N. E. Steenrod, Products of cocycles and extension of mappings, Ann. of Math., 48 (1947).

Mathematical Institute, Nagoya University 